\title{
Políticas educativas en Corea del Sur: buenas prácticas TIC en la sociedad del conocimiento*
}

DOI: $10.32870 /$ mycp.v14i40.361

Alicia González Pérez ${ }^{1}$

\section{Resumen}

$\mathrm{E}$ $n$ este artículo se trata de dar a conocer cómo está conformado el sistema educativo coreano actualmente, así como el valor formativo que las tecnologías de la información y la comunicación (TIC) están teniendo en la práctica educativa. En Corea el papel de la política está siendo fundamental para introducir las TIC en la escuela, generar procesos de innovación educativa a través de buenas prácticas educativas TIC en el aula, y formar al profesorado, como estrategias clave para integrar las TIC en los procesos de enseñanza-aprendizaje. De ahí que se destaquen algunos proyectos educativos con TIC, como por ejemplo el Proyecto EBS, Edunet y Ciber Home System, con el fin de entender cómo se han introducido las TIC en el sistema educativo coreano.

Palabras clave: políticas educativas en Corea del Sur, tecnologías de la información y la comunicación (TIC), buenas prácticas con TIC, innovación educativa.

\section{Abstract}

This article attempts to understand how the Korean educational system is configured now and the formative value that information and communication technologies (ICT) are having on educational practice. In Korea, the

* Artículo recibido el 16 d e agosto de 2010 y dictaminado el 16 de septiembre de 2010.

1. Profesora del Departamento de Didáctica y Organización Educativa de la Facultad de Educación de la Universidad de Sevilla, España. Correo electrónico: aliciagp@us.es 
role of the government has been essential to introduce ICT in schools, to generate innovative processes through good practices in education with ICT in classrooms and to train teachers as a key strategy for integrating ICT in the processes of teaching and learning. Therefore it is necessary to point out some ICT educational projects such as EBs Project, Edunet and Cyber Home System in order to understand how ICT has been introduced in the Korean educational system.

Keywords: educational policies in South Korea, information and communication technologies, good practices with ICT, educational innovation.

\section{Introducción}

A lo largo de este artículo se va a dar a conocer cómo esta conformado el sistema educativo coreano, así como el valor formativo de las Tic en la práctica educativa y la implicación social en la creación de nuevos escenarios formativos en la República de Corea del Sur.

En este trabajo se describen las políticas educativas más actuales, donde el gobierno coreano tiene como propósito crear un sistema educativo competitivo que se adapte a los nuevos tiempos y que atienda todas las áreas del fenómeno e-learning. Así, podremos entender cómo se han introducido las TIC en la educación coreana y cuáles son las perspectivas de futuro.

Actualmente, las TIC son consideradas en el ámbito educativo como agentes de cambio de las sociedades modernas, que generan nuevas formas de aprendizaje y que merecen ser estudiadas. A través de este repaso se trata de conocer las tendencias futuras que el

Actualmente, las TIC son consideradas en el ámbito educativo como agentes de cambio de las sociedades modernas, que generan nuevas formas de aprendizaje y que merecen ser estudiadas gobierno coreano impulsa para introducir las TIC en el sistema educativo y para el desarrollo de la sociedad de la información y del conocimiento.

Las principales razones que justifican el desarrollo de políticas educativas TIC no sólo se deben a los cambios acontecidos en el sistema educativo, producidos por el nuevo modelo de sociedad de la información y del conocimiento, sino también a las nuevas competencias digitales a desarrollar con los alumnos, 
a las nuevas formas de entender los procesos de enseñanza-aprendizaje y al intento de innovar en los usos de herramientas TIC para el desarrollo de competencias digitales tanto en el profesorado como en el alumnado.

\section{Evolución del sistema educativo coreano hacia la sociedad del conocimiento}

Tradicionalmente los coreanos han dado gran importancia a la educación, no sólo como un medio para la realización personal sino también como un medio de avance y reconocimiento social. Desde la teoría tradicional confucionista, el principal objetivo de la educación consistía en cultivar la moral y la ética de las personas. Sin embargo, con la industrialización la educación se fue centrando cada vez más en cómo utilizar ésta para alcanzar los objetivos económicos. Por eso se puede decir que la educación en Corea ha sufrido numerosas transformaciones y desarrollos que han hecho cambiar su objetivo para adaptarse a las necesidades de cada tiempo.

En 1945, después de la liberación del pueblo coreano, se establecieron los pilares de una educación democrática, universal y obligatoria que tuvo en cuenta los principios establecidos en la Constitución de 1948. En los años cincuenta, la Ley de Educación fue decretada y se hizo obligatoria la educación elemental a todos los ciudadanos en edad escolar. De esta forma se llevaron a cabo diversas acciones con objeto de implementar de forma escalonada la reforma escolar, además de reducir las altas tasas de analfabetismo existentes y apostar por una educación de calidad. Para ello había que poner en marcha todo un sistema de gestión educativa que abarcaría desde la creación de centros para la formación de profesorado hasta la distribución de libros de texto de primaria.

La Ley de Educación Nacional de Corea del Sur, promulgada en 1968, reconoce la necesidad de una reforma educativa que contemple los fundamentos filosóficos de la educación en pro de la identidad nacional, y del respeto a la historia y a la tradición. La sección 1 , artículo $1^{\circ}$ de la Ley de Educación Nacional, dice:

La educación tiene su base en el lema Hong-ik-in-kan [beneficiar al ser humano] y debe ayudar a todos los ciudadanos a perfeccionar el carácter de cada individuo, a desarrollar la capacidad para la vida independiente y a promover la prosperidad de todos los humanos. 
A lo largo de sus artículos, la ley enfatiza el balance necesario entre tradición y desarrollo, y entre las necesidades de los individuos y de la nación.

Desde la década de los sesenta el gobierno coreano puso especial énfasis en la educación tecnológica. La Ley de Promoción de la Educación Industrial, promulgada en 1963, financió mediante becas a investigadores, profesores y estudiantes que trabajaron y estudiaron sobre las áreas relacionadas con la industria.

Es por ello que el gobierno se ha preocupado por mantener, desde entonces, una constante en las reformas sobre educación tecnológica tanto a medio como a largo plazos. Así pues, en 1992 con la reforma de los estudios profesionales, se exigió un nivel más alto de conocimientos y habilidades para que los egresados pudieran trabajar en el sector industrial. Pero con esta reforma se daba la posibilidad de estudiar dos o tres años más y conseguir el título de técnico profesional.

Así pues, el sistema educativo de Corea del Sur contiene una serie de factores que son de interés permanente tanto para el gobierno como para la sociedad, y ha cosechado éxitos de diversa índole, como son el rápido despegue económico e industrial, y la inexistencia del analfabetismo prácticamente en toda la nación.

En la siguiente gráfica se puede ver la evolución de la demanda educativa medida en el número de alumnos que ingresaron en los niveles de primaria y secundaria desde 1945 hasta nuestros días. Es importante resaltar lo que nos muestra esta gráfica: por un lado, se ve cómo a partir de 1945 se produce una universalización de la educación primaria. Una vez que se consigue reducir esas tasas de analfabetismo al mínimo, se empieza a observar cómo en los años setenta se produce una universalización de la enseñanza secundaria.

Es a finales de los cuarenta cuando Corea establece un sistema educativo moderno que se conoce como 6-3-3-4. Esto significa que la enseñanza elemental dura seis años, la enseñanza de secundaria obligatoria tres años, la enseñanza de secundaria superior otros tres, y los estudios universitarios cuatro años. Actualmente está vigente dicho modelo de sistema educativo. Respecto a la educación infantil, se debe añadir que va de tres a seis años, y aunque no es obligatoria, ya que no hay ninguna ley que así lo regule, la mayoría de los coreanos creen que debería serlo. La educación es obligatoria hasta los 15 años, una vez terminada la secundaria obligatoria.

A continuación se muestra un esquema que intenta aclarar de forma visual cómo se estructura el sistema educativo coreano en las distintas etapas. 


\section{Figura 1}

Evolución alumnos matriculados en primaria

$$
\text { y secundaria desde } 1945 \text { a } 2002
$$

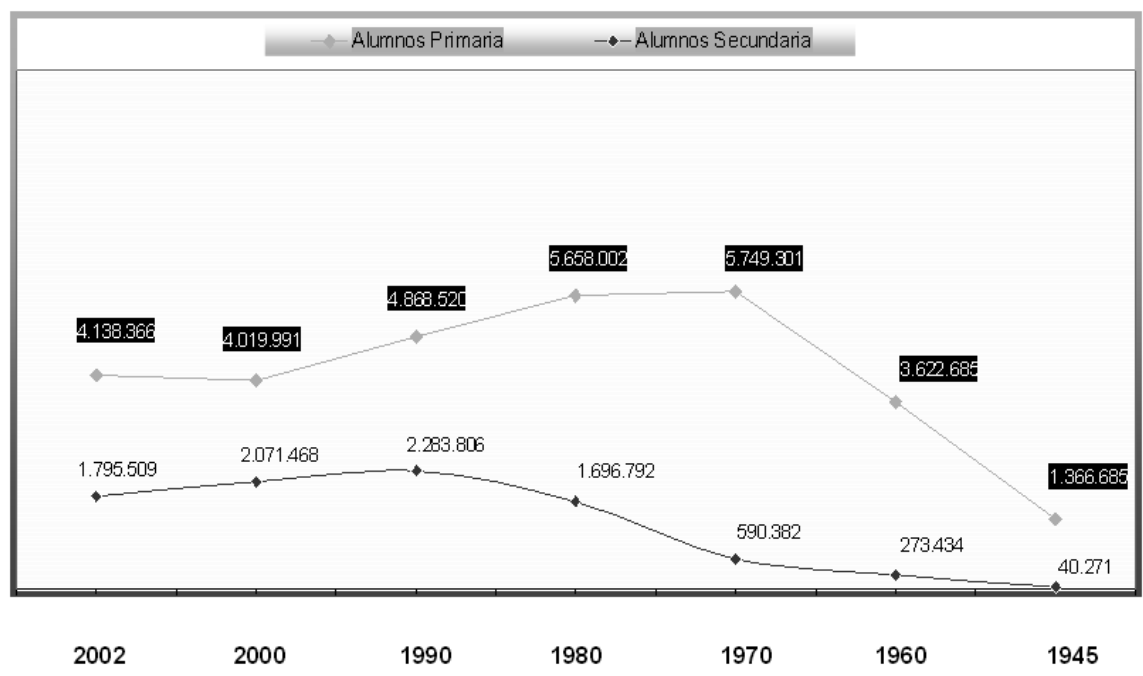

Fuente: MOE\&HRD.

Figura 2

Sistema educativo coreano

\begin{tabular}{lllllllllllllllllllllllllll}
3 & 4 & 5 & 6 & 7 & 8 & 9 & 10 & 11 & 12 & 13 & 14 & 15 & 16 & 17 & 18 & 19 & 20 & 21 & 22 & 23 & 24 & \multicolumn{1}{c}{} & 26 & 27 & 28 & 29
\end{tabular}

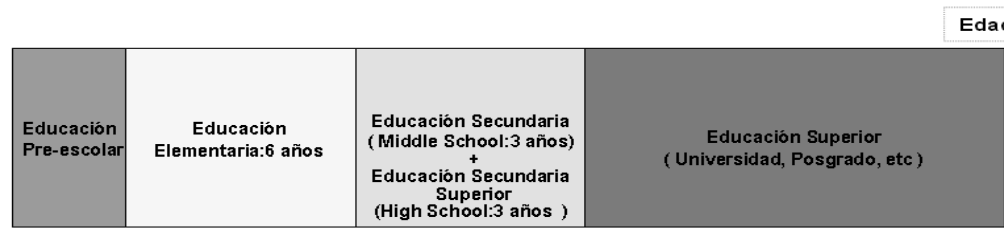

Educación Especial

Años Escolares

$\begin{array}{lllllllllllllllllllllll}1 & 2 & 3 & 4 & 5 & 6 & 7 & 8 & 9 & 10 & 11 & 12 & 13 & 14 & 15 & 16 & 17 & 18 & 19 & 20 & 21 & 22 & 23\end{array}$ 
La Ley de Educación Nacional de Corea del Sur, promulgada en 1968, fue reemplazada en 1998 por los decretos que regularizaban la educación básica, la educación primaria y secundaria, y la educación superior. El decreto de educación primaria y secundaria cubre todas las cuestiones que tienen que ver con la educación preescolar y la educación de primaria y secundaria. Sin embargo, el decreto de educación superior sólo se centra en gestionar cuestiones relativas a ésta.

En Corea del Sur existen tres tipos de escuelas: nacionales, públicas y privadas. Cuando se habla de escuelas nacionales se hace referencia a las escuelas fundadas por el Estado. La gran mayoría de las escuelas primarias pertenecen a este grupo y sólo un pequeño número de escuelas secundarias son englobadas en esta categoría. Por ejemplo, en Seúl sólo se cuenta con una escuela de secundaria nacional que está adscrita a la Facultad de Educación de la Universidad Nacional de Seúl. El Estado coreano siguió la política de fundar una universidad nacional por cada provincia; por ejemplo, la Universidad Nacional de Seúl o la Universidad Nacional de Chungcheongnamdo, entre otras.

En lo que respecta a las escuelas públicas, hay que decir que son las fundadas por los gobiernos locales. En realidad las escuelas nacionales deberían pertenecer a este grupo, pero hasta el momento se diferencian unas de otras. También existen escuelas con distintas acepciones, ya que en la ciudad de Incheon no hace mucho se fundó una escuela de secundaria pública e internacional, pero también hay escuelas de ciencias que pertenecen a este grupo.

Finalmente, hay un gran número de escuelas privadas en todos los niveles, sobre todo en los niveles no obligatorios. Por ejemplo, la mayoría de las escuelas de secundaria de idiomas extranjeros son privadas, al igual que muchas universidades, por ejemplo la Universidad de Dankook.

A continuación pasamos a conocer las políticas educativas con TIC que el gobierno coreano establece para adaptarse a la sociedad de la información y del conocimiento.

\section{El séptimo currículo nacional en Corea del Sur: hacia una sociedad del conocimiento}

Los esfuerzos para reformar la educación con el fin de introducir las TIC en el currículo, han sido una constante por parte del gobierno coreano desde los 
años ochenta. En Corea, el Ministerio de Educación, Ciencia y Tecnología ${ }^{2}$ es la máxima institución educativa encargada de desarrollar las coordenadas y directrices de las políticas educativas del país, además de establecer un marco estratégico que busque la calidad y eficacia para acceder a las Tic desde la igualdad. Un punto fuerte de la política educativa coreana ha sido la apuesta por la introducción de las TIC en la educación. Es por ello que el e-learning está siendo impulsado en todos los niveles educativos como estrategia nacional para integrar a la población en la sociedad del conocimiento y construir una comunidad educativa de aprendizaje online.

Actualmente la sociedad del conocimiento plantea nuevos retos que repercuten directamente en la forma de actuar de los profesionales de la educación. Ahora no se trata de transmitir conceptos a través de clases magistrales, sino de dar herramientas necesarias para que sea el alumno quien aprenda a gestionar la información cuando quiera, con quien quiera y desde donde quiera.

La simple dotación de infraestructuras tecnológicas no asegura el cumplimiento de los objetivos que las políticas educativas plantean, ya que la tecnología en sí misma no mejora la calidad educativa. Por ello, debemos generar competencias y destrezas entre los estudiantes, profesores y otros agentes educativos implicados en el uso pedagógico de las TiC en la enseñanza, que mejoren la calidad y eficacia de la docencia y complementen la formación en y con las TIC.

En mayo de 1995 se presentó una nueva reforma educativa que apostaba por una educación abierta orientada hacia las necesidades individuales, y una educación especializada y diversificada basada en el principio de autonomía y la responsabilidad del individuo (Jeong Won Kim, 2004). A partir de esta reforma se comenzó a gestar el séptimo currículo nacional, cuyo objetivo principal fue impulsar la sociedad del conocimiento en las instituciones escolares. Desde entonces las TIC comenzaron a ganar importancia en los planes de estudio y en la integración curricular.

El séptimo currículo nacional fue aprobado el 30 de diciembre de 1997 y supuso la aplicación, primero en la educación primaria, y seguidamente en el 2000 en la educación secundaria, hasta que se hizo extensivo en todos los niveles y etapas educativas en 2004.

2. Se conoce como Ministerio de Educación, Ciencia y Tecnología recientemente. La antigua designación de este ministerio correspondía a las siglas MOE\&HRD (Ministry of Education \& Human Resources Development). 
Finalmente y para concluir, no fue sino hasta los años noventa cuando se dibujó un marco legislativo base, con la Ley para la Promoción de las TIC, que se concretó en julio de 1996 en el I Plan de Adaptación de la Educación a la Sociedad de la Información. Así pues, en 1997 se empezó a dotar de infraestructuras a todas las escuelas de educación primaria y secundaria según lo establecido en este primer plan. En mayo de 2001 el gobierno coreano anunciaba el II Plan de Adaptación de la Educación a las TIC, el cual se centraba más en la creación de recursos educativos, dotación de los equipos informáticos y el mantenimiento de toda la infraestructura tecnológica tanto en las escuelas como en las oficinas de educación.

\section{Desarrollo de proyectos educativos con TIC en la etapa obligatoria de la educación coreana}

En la actualidad el papel de la política está siendo fundamental para introducir las TIC en la escuela. De ahí que se generen buenas prácticas educativas TIC que van a tener un alcance importante dentro de la comunidad educativa, $y$ que a continuación pasamos a explicar.

Antes es importante introducir qué entendemos por buena práctica, ya que es un concepto amplio. La noción de buena práctica tiene su origen en el ámbito empresarial y se utiliza como calificativo a cualquier actividad que ofrece buenos resultados en el contexto en el que se utiliza, por lo que la búsqueda de resultados siempre eficaces y eficientes en el ámbito referido es lo que determinó que se acuñara este término. De manera muy genérica podemos decir que la adopción del término "buenas prácticas" se hace no para determinar aquellas experiencias que pueden considerarse como la mejor actuación imaginable sobre un contexto específico, sino para sacar a la luz aquellas actuaciones que suponen una transformación en las formas y procesos de actuación y que pueden suponer el germen de un cambio positivo en los métodos de actuación tradicionales (De Pablos y González, 2007).

A continuación se presentan algunas iniciativas innovadoras con TIC desarrolladas por el gobierno coreano, que tratan de introducir las TIC en su sistema educativo de forma eficiente. Éstas son: 
Proyecto EBS ${ }^{3}$ (Education Broadcasting System).

El Proyecto EBS fue lanzado por el MOE\&HRD el 1 de abril de 2004 y nació con el objetivo de paliar las diferencias educativas que se estaban generando entre estudiantes. Es decir, se observa un aumento considerable en cuanto a inversión en clases privadas, lo que genera una brecha educativa muy fuerte entre estudiantes coreanos. Pero también esta iniciativa es una apuesta clara por una formación permanente de la sociedad coreana.

El sistema educativo coreano se caracteriza por ser muy competitivo y esto lleva a los coreanos a esforzarse mucho para acceder a las mejores universidades de su país, ya que ello constituye un símbolo de prestigio social para los coreanos. De ahí que inviertan mucho tiempo y dinero en prepararse para la prueba de acceso a la universidad, que se caracteriza por ser muy exigente. Esta situación lleva a muchos jóvenes coreanos a salir al extranjero con el fin de ver reconocido su esfuerzo académico, debido a que estudiar en el extranjero es el camino paralelo para conseguir lo que no han podido alcanzar en su país. De ahí que se desarrollen actuaciones políticas para evitar esta brecha formativa.

El EBS consta de una infraestructura de retransmisión pública que incluye servicios de televisión, radio e Internet vía satélite, que proporcionan no sólo contenidos educativos sino también cursos de preparación del examen de acceso a la universidad (CSAT). El EBS parte de la premisa de que todos los ciudadanos tienen igual derecho a la educación, y trata de romper las diferencias de edad, género y clase.

El Proyecto EBS fue decisivo para introducir de forma generalizada la educación online como un nuevo método de enseñanza/aprendizaje entre la población coreana y la creación de un contexto de aprendizaje virtual para todos, que rompió las barreras espacio-temporales.

Pocos meses después se pudo observar el impacto que dicha política había tenido en la población coreana. En agosto de 2005 el número de usuarios registrados en la plataforma educativa rondaba alrededor de 1.8 millones de usuarios y la media de descargas vOD era de 111,024 archivos. Todos los días se descargaban una media de 33,918 archivos de video streaming. El total de descargas hasta el momento ascendía a 8'649,050.

3. Sistema de Transmisión Educativa (Education Broadcasting System). 
Edunet: Centro Nacional de Enseñanza/aprendizaje Online

Edunet es un servicio de información educativa que permite a todos los ciudadanos, incluyendo estudiantes, profesores, y público en general acceder a una valiosa información sobre temas educativos, y de establecer una comunidad educativa online. Además también favorece la creación de comunidades de aprendizaje e intereses generales, lo cual facilita el intercambio de experiencias educativas y el intercambio de información. El profesorado puede colaborar con otros profesores de distinta procedencia y establecer formas de trabajo cooperativas, así como intercambiar materiales y recursos educativos. Esta nueva forma de trabajo colaborativo hizo que en 2003 80\% de profesores participaran en dicho servicio.

Su uso es sencillo y fácil, y una vez que el usuario está registrado en la plataforma puede acceder a todos los servicios y recursos online de forma gratuita. También se puede hacer miembro de cualquier comunidad educativa existente e incluso crear la suya propia. Edunet es un servicio que sobre todo trata de favorecer la formación permanente de los coreanos.

Gracias a todas estas iniciativas se observa que al generalizarse el uso de Internet en la enseñanza, se produce un cambio en la forma de enseñar, pasándose hacia metodologías de colaboración grupal que maximizan los usos pedagógicos de Internet. El trabajo en equipo se normaliza, permitiendo tanto a profesores como alumnos participar fuera de los límites espacio-temporales que caracterizan a la clase tradicional y les da la oportunidad de trabajar en forma interactiva.

\section{El sistema de aprendizaje Cyber Home (CHLS) ${ }^{4}$}

El chls se basa en un tipo de educación a través de las Tic dirigido a los estudiantes de primaria y secundaria que de forma voluntaria deciden participar en esta plataforma de aprendizaje online (Innwoo Park, 2005). El objetivo principal ha sido tratar de mejorar la calidad de la educación pública y reducir el gasto de las tutorías privadas a través de metodologías de trabajo más autónomas.

El primer proyecto piloto se lanzó en tres oficinas de educación: Daegu, Kwangju y Gyeongsangbuk-do, para generalizarse a toda Corea en 2005. En

4. Ciber Home Learning System. 


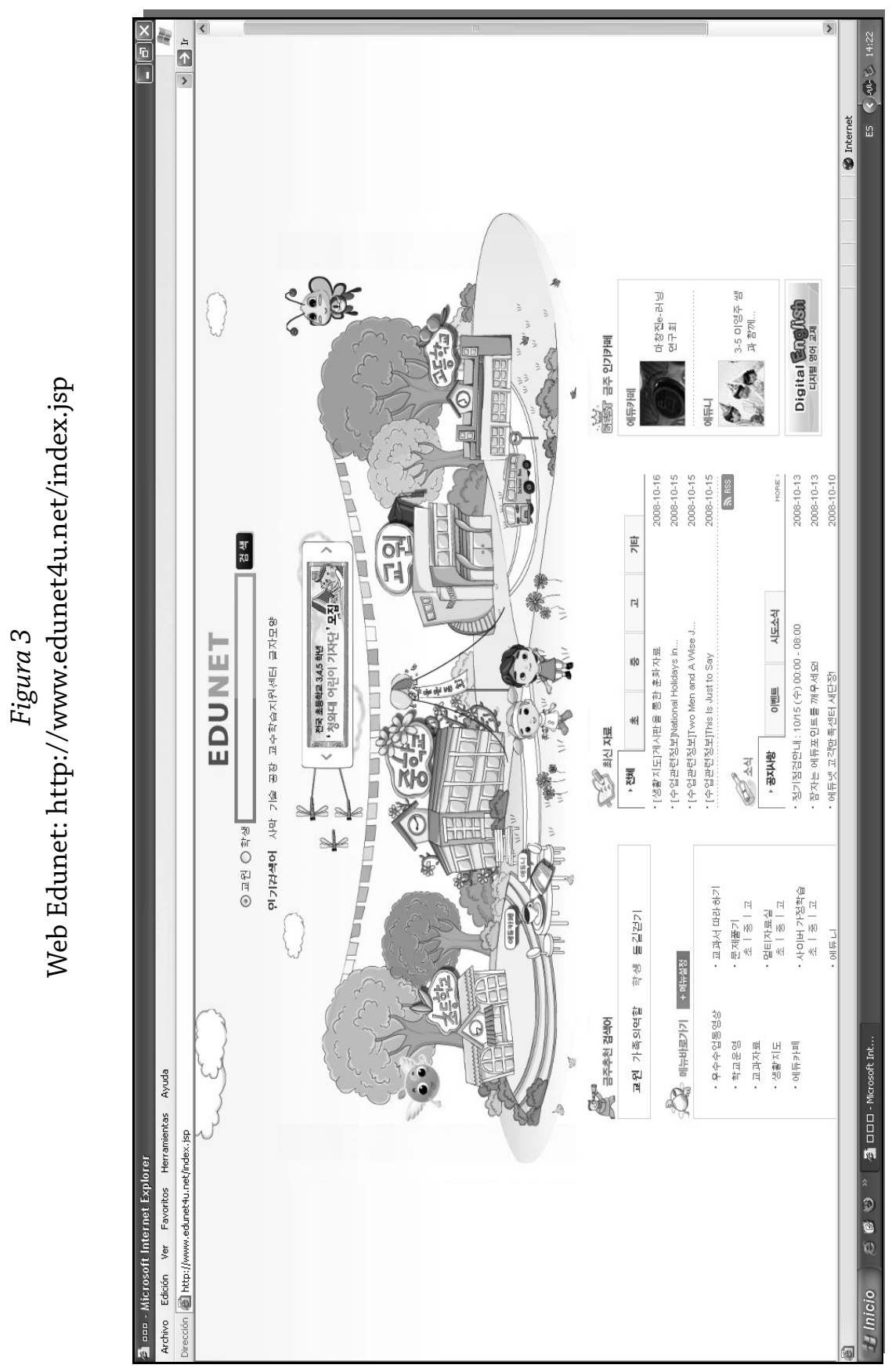


tan sólo nueve meses más de un millón de usuarios participaron en este programa, que incluía contenidos gratuitos para aprender de forma autónoma y tests online. Permitía además la posibilidad de interactuar con profesores y realizar actividades de aprendizaje. Además, se eligió a la EBS como el mejor proveedor de servicios educativos, y a las seis oficinas de educación (Daegu, Incheon, Gyeonggi-do, Gangwon-do, Chungcheongbuk-do y Jeollanm-do) como colaboradores del proyecto.

En este sistema de aprendizaje (CHLS) hay que destacar cuatro servicios principales:

- Servicio online gratuito para organizar los contenidos de aprendizaje, donde se lleva a cabo la evaluación de los contenidos de aprendizaje por nivel de dificultad, que ofrece EBS, KERIS, KICE y las oficinas de educación metropolitanas y provinciales. Además se ponen a disposición del alumnado contenidos educativos gratuitos pertenecientes al sector privado, pero sólo en las páginas que operan para las oficinas de educación provinciales y metropolitanas.

- Servicio de aptitud ciber docente, donde se gestiona una base de datos sobre preguntas acerca de asignaturas, unidades y temas, y un servicio online de autoevaluación.

- Servicio de asesoramiento donde el ciber profesor resuelve a los alumnos cuestiones sobre los contenidos educativos que se presentan en las distintas áreas de conocimiento.

- Servicio de asignación de un profesor a un grupo de alumnos para la ciberclase. Así, es posible ofrecer a los alumnos un sistema de tutorías por materias o niveles de dificultad.

Según las primeras evaluaciones llevadas a cabo por el KERIS a una muestra de 19,567 estudiantes, 1,531 profesores, 5,345 padres y 468 funcionarios que trabajaban en las cinco oficinas de educación (Busan, Gangwon-do, Gyeongsangbuk-do, Chungcheongnam-do y Jeollanam-do) acerca de la efectividad de este sistema, se puede concluir lo siguiente de acuerdo con Sang-Hyun Jang (2006):

Primero. Según la evaluación de los estudiantes de los diferentes niveles, el CHLS resulta satisfactorio y eficaz, en orden de preferencia, para la educación básica, luego para la educación secundaria obligatoria y en menor medida para la educación de secundaria superior. Posiblemente esto es debido a que 
la mayoría de los contenidos son archivos flash y animaciones, y esto puede resultar mucho más atractivo para estudiantes de educación básica. De modo que sería necesario establecer otras medidas que atraigan a los estudiantes de educación secundaria superior, como por ejemplo proporcionar contenidos y tests, dependiendo del perfil del estudiante.

Segundo. Se ha evaluado la influencia de este sistema de aprendizaje en distintos contextos sociales, teniendo en cuenta las diversas áreas residenciales y el nivel de ingresos, entre otros factores contextuales. De este análisis se deduce que los estudiantes que provienen de familias con altos ingresos y que viven en áreas metropolitanas, alcanzan un nivel de satisfacción más alto, que condiciona de forma positiva el nivel de logros. Sin embargo, las familias con bajos ingresos tienen más dificultades de acceso a las Tic, lo cual es razón suficiente para que el cHLS sea menos eficaz. También cabría destacar que los residentes en pequeñas ciudades y pueblos, en general, tienen más dificultades de acceso a la educación privada, y por tanto tienen mayores expectativas respecto a este sistema.

Tercero. Se analiza la relación entre eficacia del chLs y el número de trimestres en que esta plataforma es utilizada. Se deduce que entre más semestres se use la plataforma, mayor es el nivel de satisfacción. Los estudiantes que más usan CHLS, mejor se adaptan a los espacios virtuales de aprendizaje y son más autónomos, con lo que desarrollan otras competencias. Por tanto, se considera importante efectuar un esfuerzo para buscar formas que desarrollen la capacidad de aprendizaje autónomo a través de métodos de aprendizaje en el ciberespacio y llevando a cabo prácticas en cHLS.

Cuarto. Destaca que un factor de éxito de chls se debe a que los estudiantes con mejores resultados académicos tienden a estudiar de forma más autónoma, de modo que se adaptan muy bien a esta metodología de aprendizaje.

Quinto. Se ha demostrado que los alumnos que voluntariamente se suscriben a la plataforma se sienten más motivados y satisfechos.

Sexto. En cuanto a la organización de clases en cHLs, los estudiantes de educación básica demuestran un grado de satisfacción mayor cuando las ciberclases van en paralelo con las clases tradicionales, mientras que los estudiantes de educación secundaria se sienten mejor cuando se les asigna grupos por regiones. Estos resultados sugieren que las actividades por regiones tienen un efecto positivo en los estudiantes de secundaria.

Séptimo. Se ha comprobado que este sistema de aprendizaje es más efectivo en familias con bajos ingresos que en familias que viven en zonas residenciales. 
Por ello es mucho más probable que las familias con bajos ingresos sustituyan las clases privadas, para trabajar en la plataforma educativa.

Para concluir, añadimos que los estudiantes sienten que CHLS fue una herramienta muy valiosa para su formación. Los profesores también reconocieron que CHLS les fue muy útil para el desarrollo de competencias en TIC, para mejorar la interacción con los estudiantes y para favorecer la mejora de su autoestima. Los padres, sin embargo, empezaron a ver las TIC como algo positivo e incluso les sirvió para ponerse al día con este tema.

\section{Conclusiones}

En este articulo se hace un repaso de cómo ha ido evolucionando el sistema educativo coreano hasta el momento actual y qué iniciativas se han desarrollado para introducir de forma eficaz las TIC en el sistema educativo. Iny percibe una apuesta fuerte del gobierno coreano por fomentar el desarrollo de un marco tecnológico potente y competitivo, que tuvo repercusiones importantes en la formación de un modelo de sociedad de la información que se ha considerado como referente a nivel mundial.

La rápida difusión de Internet en todo el mundo durante la segunda mitad de los años noventa permitió visualizar por primera vez un soporte material tangible de lo que podría ser esa revolución de la información.

El fenómeno Internet catalizó también un impulso renovado, desde los poderes públicos, a propuestas de sociedad de la información que no se limitaban al desarrollo de las tecnologías o del sector TIC que las produce y comercializa, sino que abarcaban, cuanto menos nominalmente, el objetivo de potenciar el efecto beneficioso de la aplicación de esas tecnologías en todos los ámbitos de la sociedad.

La introducción de las TIC en el currículo escolar como estrategia para integrar a la población en la sociedad del conocimiento, provocó cambios en la organización y la planificación de los procesos de enseñanza/aprendizaje.

\section{Referencias bibliográficas}

De Pablos Pons, J., y R. Jiménez Cortés, “Buenas prácticas con TIC apoyadas en las políticas educativas: Claves conceptuales y derivaciones para la formación en competencias ECTS", Relatec, vol. 6, núm. 2, 2007, pp. 15-28. 
De Pablos Pons, J., y T. González Ramírez, Politicas educativas e innovación educativa apoyadas en TIC: Sus desarrollos en el ámbito autonómico, II Jornadas Internacionales sobre Políticas Educativas para la Sociedad del Conocimiento, 7-10 de marzo de 2007, Granada.

González Pérez, A., “¿Qué nos interesa evaluar de las políticas educativas TiC españolas?”, Fuentes, vol. 10, Universidad de Sevilla, Sevilla, 2010, pp. 206-220. http://www.revistafuentes.es/index0.php

- - "El bienestar emocional del profesorado en los centros TIC como factor de innovación educativa", Relatec, vol. 7, núm. 2, Universidad de Extremadura, 2008, pp. 45-55. http://campusvirtual.unex.es/cala/editio/index. php?journal=relatec\&page=issue\&op=archive

-_, "La educación en Corea del Sur: Del viejo al nuevo paradigma educativo", Revista de Estudios de Asia y América, mayo, Universidad de Dankook, Seúl, 2010, pp. 25-40.

- - "Políticas educativas públicas en Corea del Sur en la formación de la sociedad de la información”, Revista Electrónica Teoría de la Educación: Educación y Cultura en la Sociedad de la Información, vol. 11, núm. 1, Universidad de Salamanca, Salamanca, 2010, pp. 406-424. http://revistatesi. usal.es/ revistas_trabajo/index.php/revistatesi/article/view/6311/6326 González Pérez, A., y Kyoung Gun Han, "E-Accessibility through Assistive Technologies for People with Disabilities in Spain, Inside European Framework", Children with Special Education Needs, vol.18, Dankook University, Seúl, Corea, 2007, pp. 47-71.

Innwoo, Park, "Cyber Home Learning System: Promise, Status and Future", The First APEC Future Education Forum, Busan, Corea, 2005.

Jeong Won Kim, "Education reform policies and classroom teaching in South Korea”, International Studies in Sociology of Education, vol. 14, núm. 2, KEDI, Seúl, 2004, pp. 125-145.

León García, M. A., "La excelencia educativa coreana: Un modelo en transición”, México y la Cuenca del Pacífico, vol. 7, núm. 22, 2004, pp. 33-39.

Richards, C., "From old to new learning: Global imperatives, exemplary Asian dilemmas and ICT as a key to cultural change in education", Globalisation, Societies and Education, vol.2, núm.3, 2004, pp. 337-353.

Sang-Hyun Jang, "Analysis on the Effectiveness of Cyber Home Learning System”, KERIS, Corea del Sur, 2006. 


\section{Páginas web de interés}

EBS, Corea del Sur, fuente contrastable en: http://www.ebs.co.kr/index.jsp

Edunet, Corea del Sur, fuente contrastable en: http://www.edunet4u.net/ index.jsp

Ministerio de Educación, Ciencia y Tecnología, Corea del Sur, fuente contrastable en: http://english.moe.go.kr 